VoL. 57 (1998) [49-54]

\title{
REMARKS ON JAMES'S DISTORTION THEOREMS
}

\author{
Patrick IN. Dowling, Narcisse Randrianantoanina and Barry TURetT
}

If a Banach space $X$ contains a complemented subspace isomorphic to $c_{0}$ (respectively, $\ell^{1}$ ), then $X$ contains complemented almost isometric copies of $c_{0}$ (respectively, $\ell^{1}$ ). If a Banach space $X$ is such that $X^{*}$ contains a subspace isomorphic to $L^{1}[0,1]$ (respectively, $\ell^{\infty}$ ), then $X^{*}$ contains almost isometric copies of $L^{1}[0,1]$ (respectively, $\ell^{\infty}$ ).

In [3], James proved that if a Banach space contains a subspace which is isomorphic to $c_{0}$ (respectively, $\ell^{1}$ ), then it contains almost isometric copies of $c_{0}$ (respectively, $\ell^{1}$ ). In this short note we shall prove complemented versions of these results and show that a dual Banach space containing a subspace isomorphic to $L^{1}[0,1]$ (respectively, $\ell^{\infty}$ ) must contain almost isometric copies of $L^{1}[0,1]$ (respectively, $\ell^{\infty}$ ). In particular, the $L^{1}[0,1]$ result is in sharp contrast to a result of Lindenstrauss and Pełczyński [4], who show that $L^{1}[0,1]$ is arbitrarily distortable, and so $L^{1}[0,1]$ can be equivalently renormed so as not to contain almost isometric copies of $L^{1}[0,1]$ (with its usual norm). As for the $\ell^{\infty}$ result, it is known that if a Banach space contains a subspace isomorphic to $\ell^{\infty}$ then it must contain almost isometric copies of $\ell^{\infty}$. This result was proved by Partington in [6]. Unaware of Partington's result, Hudzik and Mastyło [2] reproved this result in the setting of function spaces.

\section{The Results}

Two Banach spaces $X$ and $Y$ are said to be $\lambda$-isometric (with $\lambda \geqslant 1$ ), if there exists a linear isomorphism $T: X \rightarrow Y$ so that $\|T\|\left\|T^{-1}\right\| \leqslant \lambda$. A Banach space $X$ is said to contain almost isometric copies of the Banach space $Y$ if, for each $\varepsilon>0$, there exists a subspace $Z$ of $X$ so that $Y$ and $Z$ are $(1+\varepsilon)$-isometric.

PRoposition 1 . If $X$ is a Banach space which contains a complemented subspace isomorphic to $c_{0}$, then $X$ contains complemented almost isometric copies of $c_{0}$.

Proof: Let $Y$ be a complemented subspace of $X$ which is isomorphic to $c_{0}$. Let $P$ be a bounded linear projection from $X$ onto $Y$.

Received 6th February, 1997

The third author was supported in part by an Oakland University Research Fellowship. The authors wish to thank Joe Diestel for bringing Partington's paper [6] to their attention.

Copyright Clearance Centre, Inc. Serial-fee code: 0004-9729/98 \$A2.00+0.00. 
Let $\varepsilon>0$ be given. Since $Y$ contains a subspace isomorphic to $c_{0}, Y$ contains a subspace $Z$ so that $Z$ and $c_{0}$ are $(1+\varepsilon)$-isometric by the James Distortion Theorem $[\mathbf{1}, \mathbf{3}, \mathbf{5}]$. Since $Y$ is a separable Banach space and $Z$ is a subspace of $Y$ isomorphic to $c_{0}, Z$ is complemented in $Y$ by Sobczyk's Theorem $[1,7]$. Let $Q$ be a bounded linear projection of $Y$ onto $Z$. Then $Q P$ is a bounded linear projection of $X$ onto $Z$. This completes the proof.

THEOREM 2. If $X$ is a Banach space which contains a complemented subspace isomorphic to $\ell^{1}$, then $X$ contains complemented almost isometric copies of $\ell^{1}$.

Proof: Let $Y_{1}$ be a complemented subspace of $X$ such that $Y_{1}$ is isomorphic to $\ell^{1}$. Let $P$ be a linear projection from $X$ onto $Y_{1}$.

Let $\varepsilon>0$ be given. Let $T: \ell^{1} \rightarrow Y_{1}$ be a bounded linear isomorphism of $\ell^{1}$ onto $Y_{1}$ and, for each $n \in \mathbb{N}$, let $x_{n}=T\left(e_{n}\right)$ where $e_{n}$ is the usual $n^{\text {th }}$ unit basis vector of $\ell^{1}$. Then, for each element $\left(a_{n}\right)_{n} \in \ell^{1}$, we have

$$
\frac{1}{\left\|T^{-1}\right\|} \sum_{n}\left|a_{n}\right| \leqslant\left\|\sum_{n} a_{n} x_{n}\right\| \leqslant\|T\| \sum_{n}\left|a_{n}\right| .
$$

For each $n \in \mathbb{N}$, define

$$
D_{n}=\left\{\sum_{k=n}^{l} a_{k} x_{k}: l \geqslant n \text { and } \sum_{k=n}^{l}\left|a_{k}\right|=1\right\} .
$$

Let $K_{n}=\inf \left\{\|x\|: x \in D_{n}\right\}$. Then $K_{n} \leqslant K_{n+1}$ and $1 /\left\|T^{-1}\right\| \leqslant K_{n} \leqslant\|T\|$ for all $n \in \mathbb{N}$. Therefore $\lim _{n \rightarrow \infty} K_{n}$ exists. Let $K=\lim _{n \rightarrow \infty} K_{n}$ and note that $K_{n} \leqslant K$ for all $n \in \mathbb{N}$. Choose $N \in \mathbb{N}$ so that $K_{n}>(1+\varepsilon)^{-1 / 2} K$ for all $n \geqslant N$. Choose $y_{1} \in D_{N}$ so that $\left\|y_{1}\right\|<(1+\varepsilon)^{1 / 2} K$. Since $y_{1} \in D_{N}, y_{1}$ can be written as

$$
y_{1}=\sum_{k=N}^{n_{1}} a_{k}^{(1)} x_{k}, \text { where } n_{1} \geqslant N \text { and } \sum_{k=N}^{n_{1}}\left|a_{k}^{(1)}\right|=1 .
$$

Choose $y_{2} \in D_{n_{1}+1}$ so that $\left\|y_{2}\right\|<(1+\varepsilon)^{1 / 2} K$. Since $y_{2} \in D_{n_{1}+1}, y_{2}$ can be written as

$$
y_{2}=\sum_{k=n_{1}+1}^{n_{2}} a_{k}^{(2)} x_{k}, \text { where } n_{2} \geqslant n_{1}+1 \text { and } \sum_{k=n_{1}+1}^{n_{2}}\left|a_{k}^{(2)}\right|=1 \text {. }
$$

Continuing in this manner, we can choose $y_{j} \in D_{n_{j-1}+1}$ so that $\left\|y_{j}\right\|<(1+\varepsilon)^{1 / 2} K$ and $y_{j}$ can be written as

$$
y_{j}=\sum_{k=n_{j-1}+1}^{n_{j}} a_{k}^{(j)} x_{k}, \text { where } n_{j} \geqslant n_{j-1}+1 \text { and } \sum_{k=n_{j-1}+1}^{n_{j}}\left|a_{k}^{(j)}\right|=1,
$$


where $n_{0}=N-1$.

For scalars $t_{1}, t_{2}, \ldots, t_{p}$ with $\sum_{j=1}^{p}\left|t_{j}\right|=1$, we have

$$
\left\|\sum_{j=1}^{p} t_{j} y_{j}\right\|=\left\|\sum_{j=1}^{p} \sum_{k=n_{j-1}+1}^{n_{j}} t_{j} a_{k}^{(j)} x_{k}\right\| \geqslant K_{N}>(1+\varepsilon)^{-1 / 2} K
$$

since $\sum_{j=1}^{p} \sum_{k=n_{j-1}+1}^{n_{j}} t_{j} a_{k}^{(j)} x_{k} \in D_{N}$.

On the other hand,

$$
\left\|\sum_{j=1}^{p} t_{j} y_{j}\right\| \leqslant \sum_{j=1}^{p}\left|t_{j}\right|\left\|y_{j}\right\|<\sum_{j=1}^{p}\left|t_{j}\right|(1+\varepsilon)^{1 / 2} K=(1+\varepsilon)^{1 / 2} K .
$$

Thus for any scalars $t_{1}, t_{2}, \ldots, t_{p}$, we have

$$
K(1+\varepsilon)^{-1 / 2} \sum_{j=1}^{p}\left|t_{j}\right| \leqslant\left\|\sum_{j=1}^{p} t_{j} y_{j}\right\| \leqslant K(1+\varepsilon)^{1 / 2} \sum_{j=1}^{p}\left|t_{j}\right|
$$

Hence the Banach space $Y=\overline{\operatorname{span}}\left\{y_{j}\right\}_{j=1}^{\infty}$ is a subspace of $Y_{1}$, and $Y$ and $\ell^{1}$ are $(1+\varepsilon)$-isometric.

Now let $z_{j}=\sum_{k=n_{j-1}+1}^{n_{j}} a_{k}^{(j)} e_{k}$ for each $j \in \mathbb{N}$, where $n_{0}=N-1$. Let $Z=$ $\overline{\operatorname{span}}\left\{z_{j}\right\}_{j=1}^{\infty}$. Since $\left(z_{j}\right)_{j}$ is a block basic subsequence of $\left(e_{n}\right)_{n}, Z$ is a subspace of $\ell^{1}$ which is isomorphic to $\ell^{1}$ and complemented in $\ell^{1}$ by a norm 1 projection $Q$. Note also that the restriction of $T$ to $Z,\left.T\right|_{Z}$, is an isomorphism of $Z$ onto $Y$. Thus we have the following diagram:

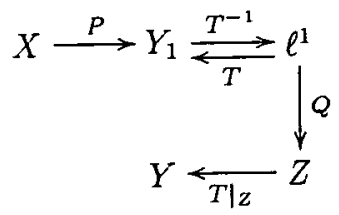

Clearly, $Y$ is complemented in $X$ by the projection $\left.T\right|_{z} Q T^{-1} P$. This completes the proof.

REMARK. Much of the proof of Theorem 2 is similar to James's original proof [3], and the proof used in Theorem 2 could also be used to prove Proposition 1. The next result is a special case of a theorem of Partington [6]. 
Corollary 3 . If $X$ is a Banach space such that $X^{*}$ contains a subspace isomorphic to $\ell^{\infty}$, then $X^{*}$ contains almost isometric copies of $\ell^{\infty}$.

Proof: If $X^{*}$ contains a subspace isomorphic to $\ell^{\infty}$, then $X$ contains a complemented subspace isomorphic to $\ell^{1}$ [1]. By Theorem $2, X$ contains complemented almost isometric copies of $\ell^{1}$. Thus for each $\varepsilon>0$, there is a complemented subspace $Y$ of $X$ so that $Y$ and $\ell^{1}$ are $(1+\varepsilon)$-isometric. Thus $Y^{*}$ and $\ell^{\infty}$ are $(1+\varepsilon)$-isometric, and since $Y$ is complemented in $X, Y^{*}$ is isometric to a subspace of $X^{*}$. This completes the proof.

THEOREM 4. If $X$ is a Banach space such that $X^{*}$ contains a subspace isomorphic to $L^{1}[0,1]$, then $X^{*}$ contains almost isometric copies of $L^{1}[0,1]$.

Proof: Let $\varepsilon>0$ be given. Since $X^{*}$ contains a subspace isomorphic to $L^{1}[0,1], X$ contains a subspace isomorphic to $\ell^{1}[\mathbf{1}]$. Hence, by the James Distortion Theorem $[1,3,5], X$ contains a subspace $Y$ so that $Y$ and $\ell^{1}$ are $(1+\varepsilon)$-isometric. Hence $Y^{*}$ and $\ell^{\infty}$ are $(1+\varepsilon)$-isometric. Since $\ell^{\infty}$ contains a subspace which is isometric to $L^{1}[0,1]$, there is an isomorphism $I: L^{1}[0,1] \rightarrow Y^{*}$ with $\|I\|\left\|I^{-1}\right\| \leqslant 1+\varepsilon$. Without loss of generality we can and do assume that $\|I\|=1$ and $\left\|I^{-1}\right\| \leqslant 1+\varepsilon$.

Let $i: Y \rightarrow X$ be the natural inclusion map. Taking an adjoint gives the following diagram:

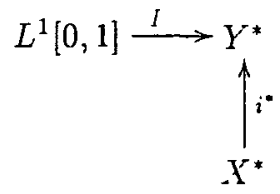

Taking adjoints again and using the fact that $L^{\infty}[0,1]$ is an injective space, there exists a bounded linear mapping $S: X^{* *} \rightarrow L^{\infty}[0,1]$ so that $\|S\|=\left\|I^{*}\right\|=1$ and the following diagram commutes:

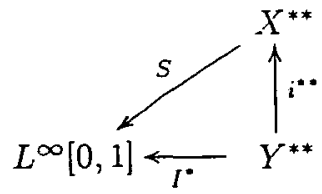

Taking adjoints yet again, we get another commutative diagram:

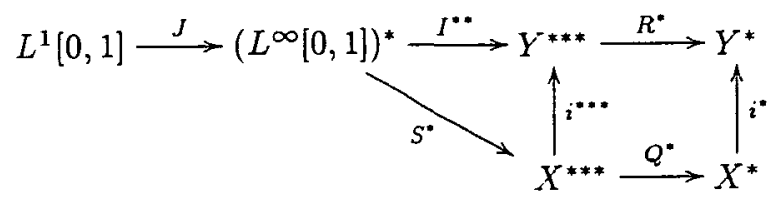

where $Q$ and $R$ denote the canonical mappings from $X$ and $Y$ to $X^{* *}$ and $Y^{* *}$ (respectively) and the mapping $J$ is the canonical mapping from $L^{1}[0,1]$ into $\left(L^{1}[0,1]\right)^{* *}=$ $\left(L^{\infty}[0,1]\right)^{*}$. 
Let $U=Q^{*} S^{*} J$. Then $U: L^{1}[0,1] \rightarrow X^{*}$ and since $\left\|i^{*}\right\|=1$ and $I^{* *}=I$ on $L^{1}[0,1]$, we have for each $f \in L^{1}[0,1]$

$$
\begin{aligned}
\|U f\| & =\left\|Q^{*} S^{*} J f\right\| \\
& \geqslant\left\|i^{*} Q^{*} S^{*} J f\right\| \\
& =\left\|R^{*} I^{* *} J f\right\| \\
& =\|I f\| \\
& \geqslant(1+\varepsilon)^{-1}\|f\|_{1} .
\end{aligned}
$$

On the other hand,

$$
\begin{aligned}
\|U f\| & =\left\|Q^{*} S^{*} J f\right\| \\
& \leqslant\left\|Q^{*}\right\|\left\|S^{*}\right\|\|J\|\|f\|_{1} \\
& =\|f\|_{1} .
\end{aligned}
$$

Thus $U\left(L^{1}[0,1]\right)$ is a subspace of $X^{*}$ so that $U\left(L^{1}[0,1]\right)$ and $L^{1}[0,1]$ are $(1+\varepsilon)$ isometric. This completes the proof.

REMARK. The proof of Theorem 4 can also be used to show that if a Banach space $X$ contains a subspace isomorphic to $\ell^{1}$, then $X^{*}$ contains almost isometric copies of $\ell^{1}\left(2^{\mathbb{N}}\right)$.

\section{REFERENCES}

[1] J. Diestel, Sequences and series in Banach spaces, Graduate Texts in Mathematics 92 (Springer-Verlag, New York, 1984).

[2] H. Hudzik and M. Mastyło, 'Almost isometric copies of $\ell_{\infty}$ in some Banach spaces', Proc. Amer. Math. Soc. 119 (1993), 209-215.

[3] R.C. James, 'Uniformly non-square Banach spaces', Ann. of Math. 80 (1964), 542-550.

[4] J. Lindenstrauss and A. Pelczyński, 'Contributions to the theory of the classical Banach spaces', J. Funct. Anal. 8 (1971), 225-249.

[5] J. Lindenstrauss and L. Tzafriri, Classical Banach spaces I. Sequence spaces, Ergebnisse der Mathematik und Ihrer Grenzgebiete 92 (Springer-Verlag, Berlin, Heidelberg, New York, 1977).

[6] J.R. Partington, 'Subspaces of certain Banach sequence spaces', Bull. London Math. Soc. 13 (1981), 162-166.

[7] A. Sobczyk, 'Projection of the space $m$ on its subspace $c_{0}$ ', Bull. Amer. Math. Soc. 47 (1941), 938-947.

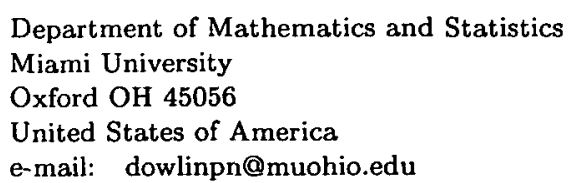

Department of Mathematics and Statistics

Miami University

United States of America

e-mail: dowlinpn@muohio.edu

\author{
Department of Mathematics and Statistics \\ Miami University \\ Oxford OH 45056 \\ United States of America \\ e-mail: randrin@muohio.edu
}


Department of Mathematical Sciences

Oakland University

Rochester MI 48309

United States of America

turett@vela.acs.oaklad.edu 